\title{
A comparison of bepridil with amiodarone in the treatment of established atrial fibrillation
}

\author{
MICHAEL S PERELMAN, WILLIAM J McKENNA, EDWARD ROWLAND, \\ DENNIS M KRIKLER
}

\begin{abstract}
From the Division of Cardiovascular Disease, Royal Postgraduate Medical School, Hammersmith Hospital, London
\end{abstract}

SUMMARY Fourteen patients with established atrial fibrillation (longer than three months) that was refractory to treatment were studied to compare the clinical and electrophysiological effects of amiodarone and bepridil. All patients initially received bepridil for three weeks (200-600 $\mathrm{mg} /$ day), followed by amiodarone for two to three months (100-400 mg/day). Bepridil seemed to be slightly more effective than amiodarone in converting the fibrillation to sinus rhythm (nine of fourteen compared with four of ten). The ventricular response in atrial fibrillation was equally well controlled by bepridil and amiodarone, both at rest and during exercise. Bepridil was associated with the development of ventricular arrhythmias in eight of fourteen patients; two had torsade de pointes, which in one degenerated into fatal ventricular fibrillation. These arrhythmias seemed to be associated with bepridil induced prolongation of the QTc interval. No ventricular arrhythmias were seen during amiodarone treatment.

Although bepridil seems to be an effective antiarrhythmic agent for the management of atrial fibrillation, its arrhythmogenic actions make it unsuitable for this purpose.

In chronic atrial fibrillation the usual therapeutic target is the slowing of the ventricular response, but a return to sinus rhythm may offer considerable additional benefits to the patient. ${ }^{1}$ Most drugs used to suppress or prevent atrial fibrillation are inhibitors of the fast inward membrane current and tend to enhance atrioventricular conduction and are therefore usually given in combination with digoxin or other agents that slow conduction in the atrioventricular node. Amiodarone combines at antiarrhythmic effect in the atria with depression of atrioventricular conduction and is therefore useful in atrial fibrillation. As bepridil, introduced as an antianginal agent, ${ }^{23}$ has been shown to have electrophysiological properties-blocking both the slow ${ }^{45}$ and fast $^{6}$ inward currents and prolonging repolarisation-it might thus be expected to have

Requests for reprints to Dr Dennis M Krikler, Division of Cardiovascular Disease, Royal Postgraduate Medical School, Hammersmith Hospital, Du Cane Road, London W12 0HS.

Accepted for publication 11 June 1987 similar actions to those of amiodarone. This study was designed to examine the antiarrhythmic potential of bepridil and compare its use in established atrial fibrillation with that of amiodarone.

\section{Patients and methods}

Fourteen patients with established atrial fibrillation of at least three months' duration and a ventricular rate of more than $100 /$ minute were studied. The protocol was approved by the ethics committee of the Royal Postgraduate Medical School and all patients gave their informed and written consent.

Because of the long half life of amiodarone, randomisation of the order of drug administration was not practical. All antiarrhythmic medications were withdrawn for at least five half lives. Patients were then given bepridil for three weeks: $300 \mathrm{mg}$ twice a day for two days then $100 \mathrm{mg}$ twice a day for five days, increasing to $200 \mathrm{mg}$ twice a day for the second week and $300 \mathrm{mg}$ twice a day for the third week. In patients who remained in atrial fibrillation there was a two week wash-out period after bepridil was stopped and before amiodarone was started. Patients 
who reverted to sinus rhythm during bepridil were followed, off all antiarrhythmic medication, after stopping bepridil. If they reverted to atrial fibrillation amiodarone was introduced at least three weeks later. In all patients, amiodarone was administered in a loading dose of $800 \mathrm{mg} /$ day for one week and then continued at $400 \mathrm{mg} /$ day for two months. In those patients who converted to sinus rhythm on amiodarone, the daily dose was further reduced to $200 \mathrm{mg} /$ day.

\section{ASSESSMENT}

Baseline rhythm and rate were assessed off medication immediately before bepridil, at the end of each week on bepridil, and after one and two months on amiodarone $400 \mathrm{mg} /$ day. In those patients receiving the reduced dose of amiodarone a further assessment was made six to eight weeks later.

At each assessment a standard procedure was followed which required two outpatient visits on consecutive days. On the first day a two channel 24 hour ambulatory electrocardiogram (Oxford Medilog 1) was recorded. When the patient returned the following day, usually in the morning and always at the same time of day, blood was drawn for the measurement of the serum drug concentration (at least 12 hours after the last tablet) and a resting 12 lead electrocardiogram was recorded. At the same visit a symptom limited treadmill exercise test (Naughton protocol) was performed during which rhythm strips were recorded every three minutes and at peak exercise.

The predominant rhythm was determined from the resting 12 lead and ambulatory electrocardiograms. The ventricular rate was recorded during 10 second rhythm strips at rest and on peak exercise. If the patient was in sinus rhythm the QT interval was measured and the $\mathrm{QT}_{\mathrm{c}}$ was derived (Bazett's formula).
The ambulatory electrocardiograms were analysed by a technician using a Reynolds Pathfinder II analysis system and checked by a physician. Rhythm variation was noted, as was the presence of ventricular arrhythmias.

\section{Results}

Table 1 shows the clinical data of the fourteen patients in the study.

In four patients bepridil was withdrawn early because of the development of arrhythmias. In one case ventricular bigeminy was present at rest, while in three patients non-sustained uniform ventricular tachycardia developed on exercise (tables 2 and 3 ).

Four patients did not go on to receive amiodarone. One patient died of a pulmonary embolus, a second died of torsade de pointes during the third week on bepridil, and two others did not complete the study.

\section{CONVERSION TO SINUS RHYTHM}

Nine of fourteen patients were converted to sinus rhythm during bepridil: two in the first week, four in the second week, and three in the third week. Atrial fibrillation reappeared in all nine patients within eight days of discontinuing bepridil.

Four of ten patients were converted to sinus rhythm during amiodarone (six of these had converted during bepridil (NS)): one by one month, two by two months, and one other after four months (maintenance dose $200 \mathrm{mg}$ daily). Two of three patients remained in sinus rhythm when the amiodarone was reduced from 400 to $200 \mathrm{mg}$ daily, while the third reverted to atrial fibrillation; sinus rhythm resumed when the dose was restored to $400 \mathrm{mg}$ daily.

Neither the cause nor the duration of atrial fibrillation were related to the successful restoration of sinus rhythm during bepridil (table 1). During

Table 1 Clinical details

\begin{tabular}{|c|c|c|c|c|c|c|}
\hline No & Sex & Age & Diagnosis & Duration of $A F$ & Bepridil & Amiodarone \\
\hline $\begin{array}{r}1 \\
2 \\
3 \\
4 \\
5 \\
6 \\
7 \\
8 \\
9 \\
10 \\
11 \\
12 \\
13 \\
14\end{array}$ & $\begin{array}{l}\mathbf{M} \\
\mathbf{M} \\
\mathbf{F} \\
\mathbf{M} \\
\mathbf{M} \\
\mathbf{F} \\
\mathbf{F} \\
\mathbf{F} \\
\mathbf{M} \\
\mathbf{F} \\
\mathbf{F} \\
\mathbf{F} \\
\mathbf{M} \\
\mathbf{M}\end{array}$ & $\begin{array}{l}54 \\
62 \\
61 \\
62 \\
58 \\
54 \\
73 \\
66 \\
68 \\
75 \\
65 \\
68 \\
66 \\
60\end{array}$ & $\begin{array}{l}\text { Pulm dis } \\
\text { DCM } \\
\text { L AF } \\
\text { L AF } \\
\text { DCM } \\
\text { Rh VHD } \\
\text { Rh VHD } \\
\text { DCM } \\
\text { DCM } \\
\text { Rh VHD } \\
\text { Rh VHD } \\
\text { Rh VHD } \\
\text { Rh VHD } \\
\text { L AF }\end{array}$ & $\begin{array}{l}\text { Several years } \\
\text { Several years } \\
3 \text { mnth } \\
1 \text { yr } \\
3 \text { mnth } \\
\text { Several years } \\
6 \text { mnth } \\
\text { Several years } \\
\text { Several years } \\
\text { Several years } \\
4 \text { mnth } \\
1 \text { yr } \\
4 \text { mnth } \\
3 \text { mnth }\end{array}$ & $\begin{array}{l}+ \\
+ \\
+ \\
+ \\
+ \\
+ \\
+ \\
+ \\
+ \\
+ \\
+ \\
+ \\
+ \\
+\end{array}$ & $\begin{array}{l}+ \\
+ \\
- \\
+ \\
+ \\
+ \\
+ \\
+ \\
+ \\
- \\
+ \\
+ \\
+\end{array}$ \\
\hline
\end{tabular}

AF, atrial fibrillation; Pulm dis, pulmonary disease; DCM, dilated cardiomyopathy; L AF, lone atrial fibrillation; Rh VHD, rheumatic valvar heart disease. 
Table 2 Rhythm during study

\begin{tabular}{|c|c|c|c|c|c|c|}
\hline \multirow[b]{3}{*}{ Patient } & \multicolumn{3}{|c|}{ Bepridil } & \multicolumn{3}{|c|}{ Amiodarone } \\
\hline & \multicolumn{3}{|c|}{$\overline{W e e k}$} & \multicolumn{3}{|c|}{ Month } \\
\hline & 1 & 2 & 3 & 1 & 2 & $3-4$ \\
\hline 1 & AF & AF & SR/AF & AF & SR & AF \\
\hline 2 & AF & - & & AF & AF & $\mathrm{AF}$ \\
\hline $\begin{array}{l}3 \\
4\end{array}$ & AF & SR & SR Died & & & \\
\hline $\begin{array}{l}4 \\
5\end{array}$ & $\begin{array}{l}\text { AF } \\
\text { SR }\end{array}$ & $\begin{array}{l}A F \\
S R\end{array}$ & $\begin{array}{l}\text { AF } \\
\text { SR }\end{array}$ & $\underset{S R}{A F}$ & $\begin{array}{l}A F \\
S R\end{array}$ & $\begin{array}{l}\text { AF } \\
\text { SR }\end{array}$ \\
\hline 6 & AF & SR & SN & AF & AF & AF \\
\hline 7 & $\mathbf{A F}$ & AF & $\mathbf{A F}$ & AF & SR & SR \\
\hline 8 & $\mathrm{AF}$ & SR & SR & - & - & - \\
\hline 9 & SR & SR & SR & AF & AF & - \\
\hline 10 & $\mathbf{A F}$ & $\mathbf{A F}$ & - & - & - & - \\
\hline 11 & $\mathbf{A F}$ & AF & SR Died & & & \\
\hline 12 & AF & $\underset{S P}{A F}$ & SR & AF & AF & $\underset{\mathbf{A F}}{\mathrm{AF}}$ \\
\hline $\begin{array}{l}13 \\
14\end{array}$ & AF & AF & SR & $\begin{array}{l}\text { AF } \\
\text { AF }\end{array}$ & $\begin{array}{l}\mathbf{A F} \\
\mathbf{A F}\end{array}$ & AF \\
\hline
\end{tabular}

AF, atrial fibrillation; $S R$, sinus rhythm; -, drug stopped.

amiodarone treatment restoration of sinus rhythm was more common in patients with a shorter duration of atrial fibrillation.

\section{CONTROL OF THE VENTRICULAR RESPONSE IN ATRIAL FIBRILLATION}

Figures 1 and 2 show the ventricular rates at rest and during exercise respectively. During bepridil treatment there was progressive reduction in the rate over the three weeks; during amiodarone treatment maximal slowing was achieved at the first assessment at one month. Maximal slowing of the ventricular rate on the two agents was similar (figs 1 and 2, left hand panel).

\section{REPOLARISATION AND VENTRICULAR} ARRHYTHMIAS

At baseline assessments no ventricular arrhythmias were detected during 24 hour ambulatory electrocardiographic monitoring. However, they were documented in eight of fourteen patients during bepridil; two of whom developed torsade de pointes (table 3). Details of these two patients follow:

A 61 year old woman with lone atrial fibrillation reverted to sinus rhythm, albeit with paroxysmal atrial fibrillation, during the second week of

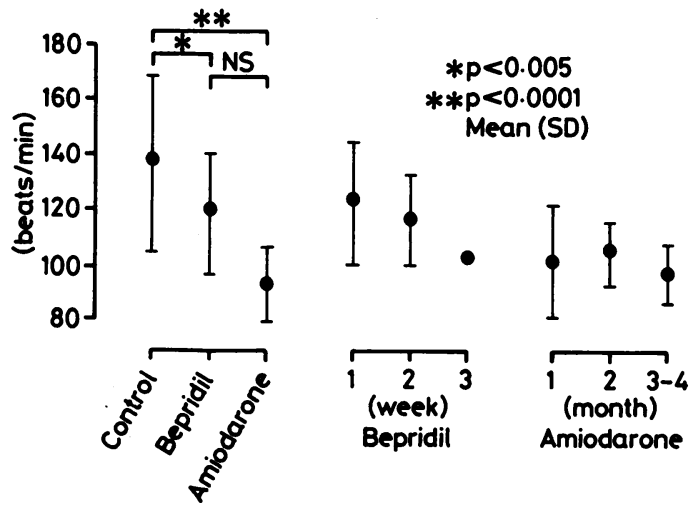

Fig 1 Plot of maximal slowing of the ventricular response rate at rest on each drug and at each assessment point in those patients in atrial fibrillation.

bepridil. While undergoing routine ambulatory monitoring, she developed torsade de pointes; immediately beforehand she had been in sinus rhythm $\left(\mathrm{QT}_{\mathrm{c}} 600 \mathrm{~ms}\right)$ with infrequent late-coupled ventricular extrasystoles, falling on the $T$ wave (fig 3). The torsade de pointes promptly degenerated into ventricular fibrillation. Necropsy showed mild left ventricular hypertrophy of uncertain aetiology; there was no clinically significant coronary artery disease.

A 65 year old woman with rheumatic mitral valve disease also developed torsade de pointes. During the third week of bepridil treatment, the electrocardiogram showed atrial fibrillation with a rapid ventricular response and runs of broad complex tachycardia which were interpreted as aberration (fig 4, 25 October 1983); the QT interval could not be determined. The next day, she reverted to sinus rhythm with a pronounced bradycardia and $a \mathrm{QT}_{\mathrm{c}}$ of $755 \mathrm{~ms}$, with brief runs of non-sustained torsade de pointes. Bepridil was discontinued and no further episodes of torsade de pointes or other ventricular arrhythmias were documented. Amiodarone was not started because she died of a pulmonary embolism

Table 3 Details of those patients who developed important ventricular arrhythmias while on bepridil

\begin{tabular}{rllll}
\hline Patient & Week & $\begin{array}{l}\text { Underlying } \\
\text { rhythm }\end{array}$ & $Q T_{\mathrm{c}}$ (ms) & Ventricular arrhythmia \\
\hline 2 & 1 & AF & & VT on exercise, VT on Holter (26 episodes) \\
3 & 3 & SR & 600 & Torsade de pointes degenerating into ventricular fibrillation \\
6 & 2 & SR & 560 & Ventricular bigeminy on rest ECG \\
8 & 2 & SR & 490 & Ventricular bigeminy on rest ECG; VT on Holter (1 episode) \\
10 & 2 & AF & & VT on exercise \\
11 & 3 & SR & VT on rest ECG and on Holter (3 episodes) \\
12 & 3 & SR & 755 & Torsade de pointes on rest ECG \\
14 & 3 & AF & 524 & Ventricular bigeminy on exercise and on Holter \\
\hline
\end{tabular}

VT, ventricular tachycardia, more than 3 complexes; Holter, 24 hour ambulatory ECG; AF, atrial fibrillation; SR, sinus rhythm. 


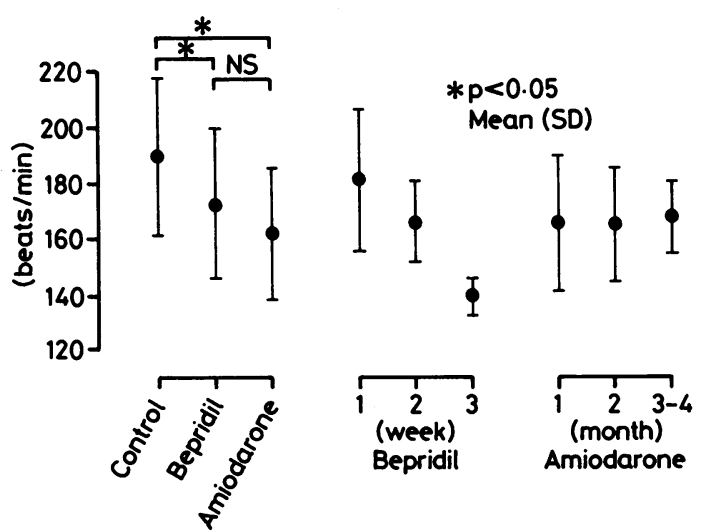

Fig 2 Plot of maximal slowing of the ventricular response rate at peak exercise on each drug and at each assessment point in those patients in atrial fibrillation.

five days after stopping bepridil, despite having been treated with anticoagulants.

Eight of the nine patients with sinus rhythm on bepridil had $\mathrm{QT}_{\mathrm{c}}$ prolongation (greater than $440 \mathrm{~ms}$ ) which was dose related (fig 5). Three developed ventricular arrhythmias, but five did not. The $\mathrm{QT}_{\mathrm{c}}$ range in the former group was 524 to 755 ms (mean (SD) $586( \pm 103))$ and in the latter group it was 408 to $521 \mathrm{~ms}(475$ ( \pm 48$)$ ) (NS). Ventricular arrhythmias developed during sinus rhythm in five and during atrial fibrillation in four; in these latter the $\mathrm{QT}_{\mathrm{c}}$ could not be derived. The two patients who developed torsade de pointes had conspicuous $\mathrm{QT}_{\mathrm{c}}$ prolongation.

\section{Discussion}

When conversion to sinus rhythm is inappropriate, or unsuccessful, atrial fibrillation is managed by slowing the rapid ventricular response. Amiodarone not only slows atrioventricular nodal conduction, but also offers the potential extra benefit of conversion to sinus rhythm, ${ }^{7}$ and there is thus an extra indication for considering its use in the management of atrial fibrillation.

Bepridil was introduced for angina ${ }^{12}$ but has a wide electrophysiological profile, affecting both fast and slow inward channels. In the guinea pig ventricle the upstroke velocity is reduced, ${ }^{4}$ while in the frog atria both slow and fast channel inhibition can be demonstrated. ${ }^{8}$ In sheep muscle fibres and Purkinje fibres the upstroke velocity is reduced in asso-
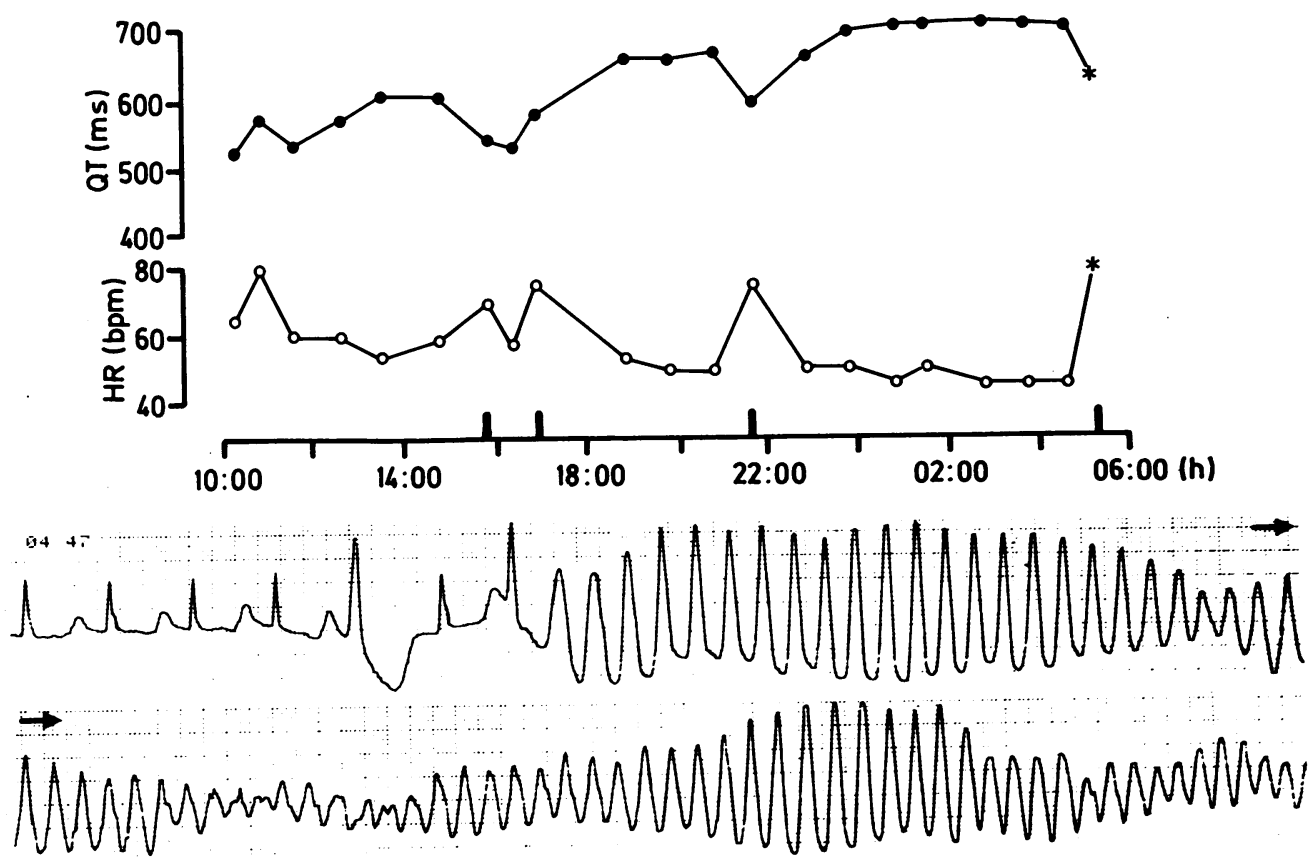

Fig 3 Plot of heart rate and QT interval (taken from ambulatory electrocardiogram) in patient 3 on bepridil ( $300 \mathrm{mg}$ twice a day). ^Initiation of the episode of torsade de pointes and ventricular fibrillation, which is shown in the printout below. 


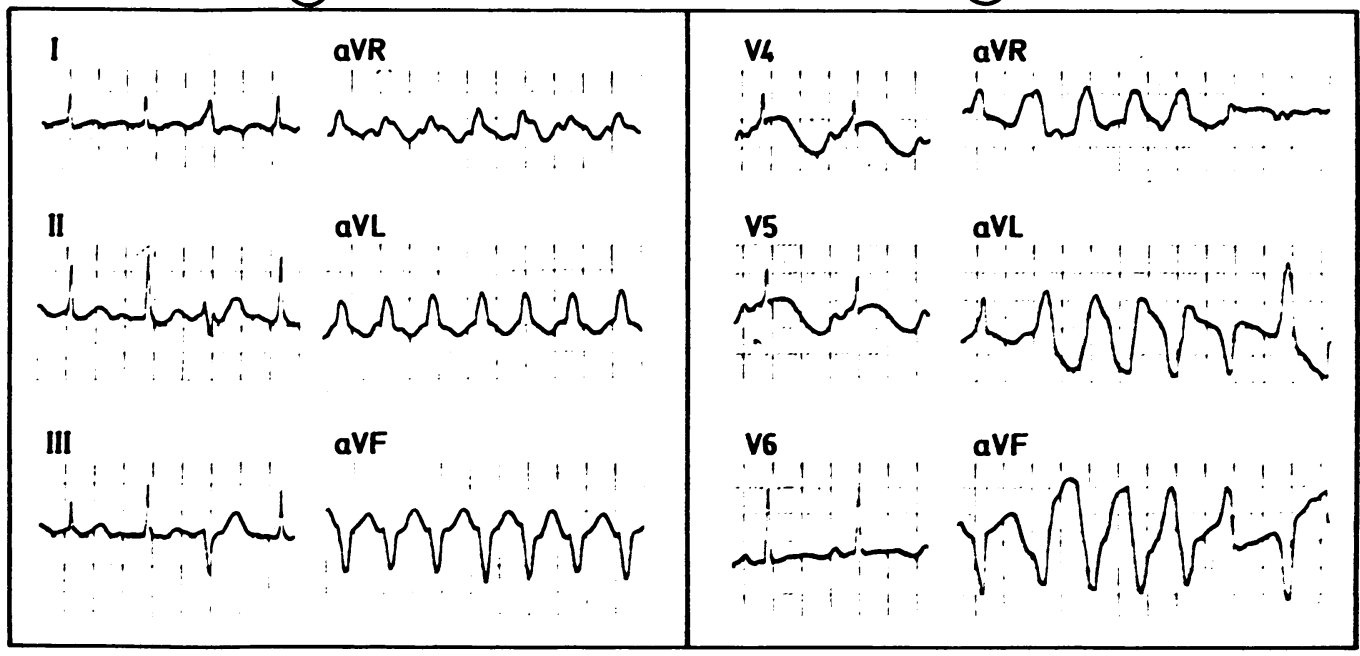

Fig 4 (a) Electrocardiogram obtained on 25 October 1983, in patient 11 showing atrial fibrillation with a rapid ventricular response (approximately 180/min). (b) Electrocardiogram on 26 October 1983 in patient 11 showing sinus rhythm (rate 95 beats/min) with considerable $Q T$, prolongation ( $755 \mathrm{~ms}$ ). In leads aVR, $a V L$, and aVF there is a brief episode of torsade de pointes.

ciation with prolongation of the action potential duration in the muscle fibres and shortening of it in the Purkinje fibres. ${ }^{6}$ It inhibits calcium flux in a similar manner to verapamil ${ }^{45}$ and increases myocardial refractoriness in many tissues. ${ }^{6}$ Studies of the electrophysiological effects of bepridil in man have shown that the increased myocardial refractoriness is most conspicuous in the atria. ${ }^{79}$

In previous studies amiodarone has been shown to convert approximately $50 \%$ of cases of atrial fibrillation to sinus rhythm, though the success rate is lower in cases of longer duration. Four of our ten patients who received amiodarone reverted to sinus

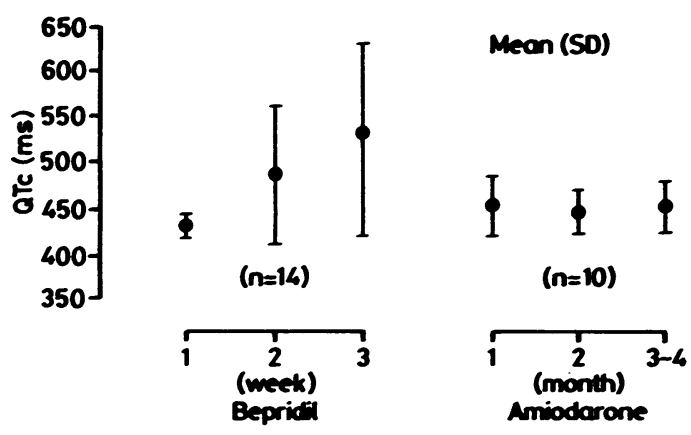

Fig 5 Plot of $Q T_{c}$ (mean $(S D)$ ) at each assessment point for those patients in sinus rhythm. rhythm, and sjx of the same ten patients were converted by bepridil; in the larger group of fourteen patients, nine reverted to sinus rhythm during the three weeks on bepridil- two in the first week, three in the second week, and four others in the third week. Thus bepridil seems to be at least as effective as amiodarone at inducing reversion.

In those patients remaining in atrial fibrillation, the effects of the two agents on the atrioventricular node, both at rest and at exercise, were equal, and very similar to the effects of digoxin and verapamil.

While bepridil has effective antiarrythmic properties, it also has significant arrhythmogenic effects. Eight of the fourteen patients developed ventricular arrhythmias while on bepridil, in two instances torsade de pointes, which in one case degenerated into ventricular fibrillation. In a review of the French experience with bepridil there were sixteen cases of torsade de pointes. ${ }^{10}$ All occurred in elderly women who had received bepridil $300 \mathrm{mg}$ per day for over eight days. In fourteen the serum potassium was $4 \mathrm{mmol} / 1$ or less, but correction of this relative hypokalaemia did not abolish the torsade de pointes.

Bepridil has a long half life of over 50 hours, and the protracted recovery from torsade de pointes suggests that the relative hypokalaemia may not have been a major contributory factor. ${ }^{10}$

The two patients in this study who developed torsade de pointes had extremely long $Q T_{c}$ intervals, 
600 and $750 \mathrm{~ms}$. There was a direct relation between $\mathrm{QT}_{c}$ and daily dose of bepridil, which may explain the arrhythmogenic effect of bepridil. The relation between dose and the QT ${ }_{c}$ has not been examined in previous studies with bepridil, but there have been abnormalities of the $T$ and $U$ waves with prolongation of the QT interval. ${ }^{311}$ In contrast, amiodarone was free of arrhythmogenic effects. The $\mathrm{QT}_{c}$ interval was prolonged but not to the same degree.

Therefore, in deciding whether bepridil offers a real advance, the benefits must be weighed against the potential risks. Bepridil is certainly a powerful antiarrhythmic agent that offers much for the management of atrial fibrillation. But the $\mathrm{QT}_{\mathrm{c}}$ prolongation and arrhythmogenic effects are very worrying, particularly as the $\mathrm{QT}_{\mathrm{c}}$ interval can only be derived with any confidence, if at all, once sinus rhythm has been achieved. In atrial fibrillation, the $\mathrm{QT}_{c}$ interval cannot be monitored as an early warning sign. Thus, in our opinion, the risks outweigh the benefits and bepridil does not offer an appreciable advantage over the established regimens or amiodarone.

\section{References}

1 Dreifus LS, Naito M, David D, Michelson EL. Atrial fibrillation: symptoms and haemodynamics. In: Kulbertus HE, Olsson SB, Schlepper M, eds. Atrial fibrillation. Mölndal, Sweden: AB Hāssle, 1983:122-32.
2 Canicave JC, Deu J, Paillet R. Un nouvel antiangoreux, le bépridil. Appréciation de son efficacité par l'epreuve d'effort au cours d'un essai à double insu contre la placébo. Thérapie 1980;35:607-12.

3 DiBianco R, Alpert J, Katz RJ, et al. Bepridil for chronic stable angina pectoris: results of a prospective multicenter, placebo controlled, dose-ranging study in 77 patients. Am J Cardiol 1984;53:35-41.

4 Vogel S, Crampton R, Sperelakis N. Blockade of myocardial slow channels by bepridil (CERM-1978). $J$ Pharmacol Exp Ther 1979;210:378-85.

5 Beaughard M, Ferrier M, Labrid C, Lamar JC, Leboeuf J, Piris P. Studies on bradycardia induced by bepridil. $\mathrm{Br} J$ Pharmacol 1982;75:293-300.

6 Kane KA, Winslow E. Antidysrhythmic and electrophysiological effects of a new antianginal agent, bepridil. J Cardiovasc Pharmacol 1980;2:193-203.

7 Rowland E, McKenna W, Holt D, Perelman MS, Krikler D. Oral amiodarone for the conversion of established atrial fibrillation [Abstract]. Circulation 1984;70(suppl II):II-439.

8 Labrid C, Grosset A, Dureng G, Mironneau J, Duchenne-Marullaz P. Some membrane interactions with bepridil, a new antianginal agent. J Pharmacol Exp Ther 1979;211:546-54.

9 Rowland E, McKenna WJ, Krikler DM. Electrophysiologic and antiarrhythmic actions of bepridilcomparison with verapamil and ajmaline for atrioventricular reentrant tachycardia. Am J Cardiol 1985;55:1513-9.

10 Leclerq JF, Kural S, Valère PE. Bépridil et torsades de pointes. Arch Mal Coeur 1983;76:341-8.

11 Dessoutter P, Haiat R. Modifications électrocardiographiques induites par le bépridil. Arch Mal Coeur 1980;73:1237-8. 